\title{
On a Lie Algebraic Characterization of Vector Bundles
}

\author{
Pierre B.A. LECOMTE, Thomas LEUTHER and Elie ZIHINDULA MUSHENGEZI \\ Institute of Mathematics, Grande Traverse 12, B-4000 Liège, Belgium \\ E-mail:plecomte@ulg.ac.be, thomas.leuther@ulg.ac.be, eliezihindula@ulg.ac.be
}

Received September 23, 2011, in final form January 23, 2012; Published online January 26, 2012

http://dx.doi.org/10.3842/SIGMA.2012.004

\begin{abstract}
We prove that a vector bundle $\pi: E \rightarrow M$ is characterized by the Lie algebra generated by all differential operators on $E$ which are eigenvectors of the Lie derivative in the direction of the Euler vector field. Our result is of Pursell-Shanks type but it is remarkable in the sense that it is the whole fibration that is characterized here. The proof relies on a theorem of [Lecomte P., J. Math. Pures Appl. (9) 60 (1981), 229-239] and inherits the same hypotheses. In particular, our characterization holds only for vector bundles of rank greater than 1.
\end{abstract}

Key words: vector bundle; algebraic characterization; Lie algebra; differential operators

2010 Mathematics Subject Classification: 13N10; 16S32; 17B65; 17B63

\section{Introduction}

Algebraic characterization of spaces goes back to Gel'fand and Kolmogorov [1], who proved in 1939 that two compact topological spaces are homeomorphic if and only if the associative commutative algebras of continuous functions growing on them are isomorphic. Since then, similar characterizations have proved useful for defining algebraically noncommutative spaces for instance.

A Lie algebra $\mathcal{D}(M)$ characterizes a manifold $M$ if for any manifold $N$, the Lie algebras $\mathcal{D}(M)$ and $\mathcal{D}(N)$ are isomorphic if and only if the manifolds $M$ and $N$ are diffeomorphic. Lie-algebraic characterization of manifolds dates back to around 1950 with a result of L.E. Pursell and M.E. Shanks [8] which states that the Lie-algebra of compactly supported vector fields on a manifold characterizes the latter manifold. Similar results have been obtained for various Lie subalgebras of $\operatorname{Vect}(M)$, the Lie algebra of vector fields on $M$.

Rather than considering Lie subalgebras of vector fields J. Grabowski and N. Poncin $[4,5,6]$ have characterized the manifold by means of the Lie algebra of linear differential operators and the Poisson algebra of fiberwise polynomial functions on the cotangent bundle. Recently, they extended, together with Kotov, their characterizations to the super context, see [2,3].

Another way of generalizing Pursell-Shanks' result is the Lie algebraic characterization of vector bundles. Let us, for instance, point out a result of P. Lecomte [7] which shows that vector bundles are isomorphic if and only if their Lie algebras of infinitesimal automorphisms are isomorphic.

In the present paper we propose a hybrid approach in the sense that we give a characterization of vector bundles in terms of a Lie algebra which is not a Lie subalgebra of vector fields. More precisely, we consider for a vector bundle $E \rightarrow M$, the Lie algebra $\mathcal{D}_{\mathcal{E}}(E)$ of homogeneous differential operators, i.e., the Lie algebra generated by all differential operators $D: \mathrm{C}^{\infty}(E) \rightarrow$ $\mathrm{C}^{\infty}(E)$ which are eigenvectors with constant eigenvalues of $L_{\mathcal{E}}$, the Lie derivative in the direction of the Euler vector field. We prove that the Lie algebra $\mathcal{D}_{\mathcal{E}}(E)$ and its Lie subalgebra $\mathcal{D}_{\mathcal{E}}^{1}(E)$ of first-order differential operators both characterize the vector bundle $E \rightarrow M$.

This new Pursell-Shanks type result is thus remarkable in the sense that it is the whole fibration that is characterized here, not only the base manifold or the total space of the vector 
bundle. However, since the proof relies on [7, Theorem 1], our characterization inherits the same hypotheses and thus holds only for vector bundles of rank $n>1$ over base manifolds $M$ whose first space $\mathrm{H}^{1}(M, \mathbb{Z} / 2)$ of the Cech cohomology vanishes.

In this work, we consider smooth manifolds which are assumed to be finite-dimensional, Hausdorff and second countable.

\section{The Lie algebra of homogeneous differential operators}

Let $\pi: E \rightarrow M$ be a vector bundle. We denote by $\mathcal{D}^{k}(E, M)$ the space of $k$-th order differential operators acting on $\mathrm{C}^{\infty}(E)$ and we define the set of homogeneous differential operators as

$$
\mathcal{D}_{\mathcal{E}}(E)=\bigcup_{k \geq 0} \mathcal{D}_{\mathcal{E}}^{k}(E)
$$

where $\mathcal{D}_{\mathcal{E}}^{k}(E)$ is generated by $\left\{T \in \mathcal{D}^{k}(E, M) \mid \exists \lambda \in \mathbb{Z}: L_{\mathcal{E}} T=\lambda T\right\}$. It follows from this definition that $\mathcal{D}_{\mathcal{E}}$ is a $\mathbb{Z}$-graded space. Moreover, $\mathcal{D}_{\mathcal{E}}$ is a Lie subalgebra of $\mathcal{D}(E, M)$. Actually, for any $T_{1}, T_{2} \in \mathcal{D}_{\mathcal{E}}(E)$ such that $L_{\mathcal{E}}\left(T_{1}\right)=\lambda T_{1}$ and $L_{\mathcal{E}}\left(T_{2}\right)=\mu T_{2}$, one has

$$
L_{\mathcal{E}}\left(\left[T_{1}, T_{2}\right]\right)=(\lambda+\mu)\left[T_{1}, T_{2}\right]
$$

An important property of $\mathcal{D}_{\mathcal{E}}(E)$ is that the following relations hold:

$$
\begin{aligned}
& \mathcal{D}_{\mathcal{E}}^{k}(E) \subset \mathcal{D}_{\mathcal{E}}^{k+1}(E), \\
& \mathcal{D}_{\mathcal{E}}^{k}(E) \mathcal{D}_{\mathcal{E}}^{l}(E) \subset \mathcal{D}_{\mathcal{E}}^{k+l}(E), \\
& {\left[\mathcal{D}_{\mathcal{E}}^{k}(E), \mathcal{D}_{\mathcal{E}}^{l}(E)\right] \subset \mathcal{D}_{\mathcal{E}}^{k+l-1}(E) .}
\end{aligned}
$$

Those relations show that, like $\mathcal{D}(E, M)$, the Lie subalgebra $\mathcal{D}_{\mathcal{E}}(E)$ is a quantum Poisson algebra $[4,5,6]$. The basic algebra of the quantum Poisson algebra $\mathcal{D}_{\mathcal{E}}(E)$ is the associative commutative algebra $\mathcal{A}(E)$ of fiberwise polynomial smooth functions on $E$, i.e.,

$$
\mathcal{D}_{\mathcal{E}}^{0}(E) \cong \mathcal{A}(E)=\oplus_{\lambda \in \mathbb{N}} \mathcal{A}^{\lambda}(E)
$$

where $\mathcal{A}^{\lambda}(E)=\left\{u \in \mathrm{C}^{\infty}(E): L_{\mathcal{\varepsilon}} u=\lambda u\right\}$. Notice that $\mathcal{A}(E) \subset \mathrm{C}^{\infty}(E)$ is preserved by the elements of $D_{\mathcal{E}}(E)$. More precisely, if $u \in \mathcal{A}^{\lambda}(E)$ and $T \in \mathcal{D}_{\mathcal{E}}(E)$ is an eigenvector of $L_{\mathcal{E}}$ corresponding to the eigenvalue $\mu$, then

$$
T(u) \in \mathcal{A}^{\lambda+\mu}(E) .
$$

In the sequel, elements of $\mathcal{D}_{\mathcal{E}}(E)$ are usually considered as endomorphisms of the vector space $\mathcal{A}(E)$.

\subsection{A local description}

Proposition 1. A linear endomorphism $T: \mathcal{A}(E) \rightarrow \mathcal{A}(E)$ is an element of $\mathcal{D}_{\mathcal{E}}(E)$ if and only if in local coordinates $\left(\left(x^{i}\right)_{1 \leq i \leq m},\left(\xi_{j}\right)_{1 \leq j \leq n}\right)$ corresponding to a local trivialization of $E$, it reads

$$
T=T^{\alpha, \beta} \partial_{\alpha} \bar{\partial}_{\beta}
$$

where $\alpha$ and $\beta$ are multi-indices, $\partial_{i}=\frac{\partial}{\partial x^{i}}, \bar{\partial}_{j}=\frac{\partial}{\partial \xi_{j}}$ and all $T^{\alpha, \beta}$ are polynomials in $\xi_{1}, \ldots, \xi_{n}$ with coefficients in $\mathrm{C}^{\infty}(M)$. 
Proof. We will prove that if $L_{\mathcal{E}} T=\lambda T$ then every $T^{\alpha, \beta}$ is homogeneous of weight $|\beta|+\lambda$, i.e.,

$$
L_{\mathcal{E}} T^{\alpha \beta}=(\lambda+|\beta|) T .
$$

Observe that for all $f \in \mathrm{C}^{\infty}(E)$, one has

$$
\left(L_{\mathcal{E}} \bar{\partial}_{i}\right) f=L_{\mathcal{E}}\left(\bar{\partial}_{i} f\right)-\bar{\partial}_{i}\left(L_{\mathcal{E}} f\right)=\sum_{j}\left(\xi^{j} \bar{\partial}_{j i} f\right)-\sum_{j}\left(\bar{\partial}_{i}\left(\xi^{j} \bar{\partial}_{j} f\right)\right)=-\bar{\partial}_{i} f
$$

so that

$$
L_{\mathcal{E}}\left(\bar{\partial}_{\beta}\right)=\sum_{r}\left(\bar{\partial}_{i_{1}} \cdots L_{\mathcal{E}} \bar{\partial}_{i_{r}} \cdots \bar{\partial}_{i_{|\beta|}}\right)=-|\beta| \bar{\partial}_{\beta}
$$

Hence, for any $u \in \mathcal{A}(E)$,

$$
\begin{aligned}
\lambda T^{\alpha, \beta} \partial_{\alpha} \bar{\partial}_{\beta} u & =L_{\mathcal{E}}\left(T^{\alpha, \beta} \partial_{\alpha} \bar{\partial}_{\beta} u\right)-T^{\alpha, \beta} \partial_{\alpha} \bar{\partial}_{\beta}\left(L_{\mathcal{E}} u\right) \\
& =\left(L_{\mathcal{E}} T^{\alpha, \beta}\right) \partial_{\alpha} \bar{\partial}_{\beta} u+T^{\alpha, \beta} L_{\mathcal{E}}\left(\partial_{\alpha} \bar{\partial}_{\beta} u\right)-T^{\alpha, \beta} \partial_{\alpha} \bar{\partial}_{\beta}\left(L_{\mathcal{E}} u\right) \\
& =\left(L_{\mathcal{E}} T^{\alpha, \beta}\right) \partial_{\alpha} \bar{\partial}_{\beta} u+T^{\alpha, \beta} L_{\mathcal{E}}\left(\partial_{\alpha} \bar{\partial}_{\beta}\right) u=L_{\mathcal{E}} T^{\alpha, \beta} \partial_{\alpha} \bar{\partial}_{\beta} u-|\beta| T^{\alpha, \beta} \partial_{\alpha} \bar{\partial}_{\beta} u .
\end{aligned}
$$

It follows that

$$
\left(L_{\mathcal{E}} T^{\alpha, \beta}-(|\beta|+\lambda) T^{\alpha, \beta}\right) \partial_{\alpha} \bar{\partial}_{\beta}=0,
$$

and the conclusion is immediate.

\section{Properties of the quantum Poisson algebra $\mathcal{D}_{\mathcal{E}}(E)$}

\subsection{A non-singular quantum Poisson algebra}

Remember that the canonical isomorphism $\mathcal{D}_{\mathcal{E}}^{0}(E) \cong \mathcal{A}(E)$ is obtained by identifying a fiberwise polynomial smooth function $u$ with the operator of multiplication $\gamma_{u}: \mathcal{A}(E) \rightarrow \mathcal{A}(E), v \mapsto u v$.

Proposition 2. The quantum Poisson algebra $\mathcal{D}_{\mathcal{E}}(E)$ is non-singular, i.e.,

$$
\mathcal{A}(E)=\left[\mathcal{D}_{\mathcal{E}}^{1}(E), \mathcal{A}(E)\right]
$$

Proof. If $u \in \mathcal{A}^{\lambda}(E)$ for some $\lambda \neq 0$, then

$$
\gamma_{u}=\left[\lambda^{-1} \mathrm{~L}_{\mathcal{E}}, \gamma(u)\right]
$$

If $u \in \mathcal{A}^{0}(E)$, then

$$
u=\sum_{i}\left[\mathrm{~L}_{X_{i}^{h}}, \gamma_{\pi^{*} v_{i}}\right]
$$

if $u=\pi^{*} v$ and $v \in \mathrm{C}^{\infty}(M)$ reads

$$
v=\sum_{i} X_{i} \cdot v_{i}
$$

with $X_{i} \in \operatorname{Vect}(M)$ and $v_{i} \in \mathrm{C}^{\infty}(M)$. Since any $v \in \mathrm{C}^{\infty}(M)$ can be decomposed in this way and since $\mathcal{D}(M)$, the space of linear differential operators of $M$, is non-singular (see $[4,5,6]$ ), the conclusion follows. 


\subsection{The (quasi-)distinguishing property}

Denote by $\mathcal{S}_{\mathcal{E}}(E)$ the set $\left\{\sigma(T): T \in \mathcal{D}_{\mathcal{E}}(E)\right\}$ where $\sigma$ stands for the principal symbol operator. This set $\mathcal{S}_{\mathcal{E}}(E)$ is a subset in

$$
\mathcal{S}(E)=\{\sigma(T): T \in \mathcal{D}(E, M)\} \cong \mathcal{A}\left(T^{*} E\right) .
$$

The Poisson bracket of $\mathcal{A}\left(T^{*} E\right)$ restricts to $\mathcal{S}_{\mathcal{E}}(E)$ and endows it with a commutative algebra structure.

The algebra $\mathcal{D}_{\mathcal{E}}(E)$ would be distinguishing in the sense of $[4,5,6]$ if for any $P \in \mathcal{S}_{\mathcal{E}}(E)$, we had

$$
(\forall u \in \mathcal{A}(E), \exists n \in \mathbb{N}: \underbrace{\{P,\{P, \ldots\{P}_{n \text { times }}, u\}\}\}=0) \Rightarrow\left(P \in \mathcal{S}_{\mathcal{E}}^{0}(E)=\mathcal{A}(E)\right) .
$$

Obviously, this is not the case. Indeed, considering the trivial bundle $\operatorname{pr}_{1}: \mathbb{R}^{2} \rightarrow \mathbb{R}$, it is easy to find a $P \in \mathcal{S}_{\mathcal{E}}\left(\mathbb{R}^{2}\right) \backslash \mathcal{S}_{\mathcal{E}}^{0}\left(\mathbb{R}^{2}\right)$ whose Hamiltonian vector field reads $H_{P}=\partial_{u^{1}}$ and for such a $P$, the right-hand side of condition (1) is trivially satisfied since for any $\{P,-\}^{n}=H_{P}^{n}=\partial_{u^{1}}^{n}$ while all elements in $\mathcal{A}_{\mathcal{E}}(E)$ are finite order polynomial in $u_{1}$.

In $[4,5,6]$, the fact that the Lie algebra of differential operators on a manifold is non-singular and distinguishing is used to obtain that any isomorphism of such Lie algebras always have two nice properties : it is filtered and its restriction to the basic algebras is an isomorphism of associative algebras. In order to obtain similar result for our Lie algebra, we need some refinement here.

In general, see $[4,5,6]$, for a quantum Poisson algebra $\mathcal{D}$ and its basic algebra $\mathcal{A}$ (whose unit is denoted by 1 ), one refers to constants for the elements of the image of the ground field $K$ by the natural embedding $k \in K \rightarrow k 1 \in \mathcal{A}$. The classical limit of $\mathcal{D}$ is the Lie algebra $S(\mathcal{D})=\oplus_{i \in \mathbb{Z}} \mathcal{D}^{i} / \mathcal{D}^{i-1}$ with the bracket given by

$$
\{\sigma(D), \sigma(T)\}= \begin{cases}\sigma([D, T]), & \text { if }[D, T] \in \mathcal{D}^{\operatorname{ord}(\mathrm{D})+\operatorname{ord}(\mathrm{T})-1} \backslash \mathcal{D}^{\operatorname{ord}(\mathrm{D})+\operatorname{ord}(\mathrm{T})-2} \\ 0, & \text { otherwise }\end{cases}
$$

where the map $\sigma: \mathcal{D} \rightarrow S(\mathcal{D})$ is the principal-symbol map while ord(D) $:=\mathrm{i}$ if $D \in \mathcal{D}^{i} \backslash \mathcal{D}^{i-1}$. Note that $\sigma(\mathcal{A})=\mathcal{A}=S_{0}(\mathcal{D})$.

Definition 1. A quantum Poisson algebra $\mathcal{D}$ with basic algebra $\mathcal{A}$ and classical limit $\mathcal{S}$ is called quasi-distinguishing if for any $P \in \mathcal{S}$,

$$
\{P, \mathcal{A}\}=0 \Rightarrow P \in \mathcal{A}
$$

We denote the centralizer of $\operatorname{ad}_{\mathcal{A}}$ in $\operatorname{Hom}_{K}(\mathcal{D}, \mathcal{D})$ by

$$
\mathcal{C}(\mathcal{D})=\left\{\psi \in \operatorname{Hom}_{K}(\mathcal{D}, \mathcal{D}): \psi([D, u])=[\psi(D), u], \forall D \in \mathcal{D}, \forall u \in \mathcal{A}\right\}
$$

Proposition 3. If $\mathcal{D}$ is non-singular and quasi-distinguishing, then

1) for any $i \in \mathbb{N},\left\{D \in \mathcal{D}:[D, \mathcal{A}] \subset \mathcal{D}^{i}\right\}=\mathcal{D}^{i+1}$;

2) any $\psi \in \mathcal{C}(\mathcal{D})$ preserves the filtration of $\mathcal{D}$;

3) for any $\psi \in \mathcal{C}(\mathcal{D})$ and any $u \in \mathcal{A}, \psi(u)=\psi(1) u$. 
Proof. The proof is similar to the corresponding one in $[4,5,6]$. We give the main steps in order to show that it is enough to assume that $\mathcal{D}$ is quasi-distinguishing.

1. If $D \in \mathcal{D} \backslash \mathcal{D}^{i+1}$ and $[D, \mathcal{A}] \subset \mathcal{D}^{i}$, then $\{\sigma(D), \mathcal{A}\}=0$. As $\mathcal{D}$ is quasi-distinguishing, $\sigma(D) \in \mathcal{A}$ and we have a contradiction since $\mathcal{A} \subset \mathcal{D}^{i+1}$.

2. We proceed by induction. For any $D \in \mathcal{D}$, we have $\psi([D, \mathcal{A}])=[\psi(D), \mathcal{A}]$. In particular, when $D \in \mathcal{A}=\mathcal{D}^{0}$, this equality reads $[\psi(D), \mathcal{A}]=0$ so that $\psi(D) \in \mathcal{A}$. Now, if $\psi\left(\mathcal{D}^{i}\right) \subset \mathcal{D}^{i}$ for some $i \in \mathbb{N}$, then for any $D \in \mathcal{D}^{i+1}$,

$$
[\psi(D), \mathcal{A}]=\psi([D, \mathcal{A}]) \in \psi\left(\mathcal{D}^{i}\right) \subset \mathcal{D}^{i} .
$$

Using point 1) of the proposition, it follows that $\psi(D) \in \mathcal{D}^{i+1}$.

3. First, note that for any $D \in \mathcal{D}^{1}$ and any $u \in \mathcal{A}$, we have $\left[D, u^{2}\right]=2 u[D, u]$. Applying $\psi$ to both sides, using point 2) of the proposition and the defining property of $\mathcal{C}(\mathcal{D})$, we get

$$
2 \psi(u[D, u])=\psi\left(\left[D, u^{2}\right]\right)=\left[\psi(D), u^{2}\right]=2 u[\psi(D), u]=2 u \psi([D, u]) .
$$

In terms of the induced derivation $\hat{D} \in \operatorname{Der}(\mathcal{A}): u \mapsto[D, u]$, we deduce that

$$
\psi(u \hat{D}(u))=u \psi(\hat{D}(u)) .
$$

In particular, for any $u, v, w \in \mathcal{A}$, we obtain

$$
\psi((u+w) v \hat{D}(u+w))=(u+w) \psi(v \hat{D}(u+w)) .
$$

The above equality reduces to

$$
\psi(u v \hat{D}(w))+\psi(w v \hat{D}(u))=u \psi(v \hat{D}(w))+w \psi(v \hat{D}(v))
$$

and for $v=\hat{D}(w)$, it reads

$$
\psi\left(u(\hat{D}(w))^{2}\right)+\psi(w \hat{D}(w) \hat{D}(u))=u \psi\left((\hat{D}(w))^{2}\right)+w \psi(\hat{D}(w) \hat{D}(u)) .
$$

As $\psi(w \hat{D}(w) \hat{D}(u))=w \psi(\hat{D}(w) \hat{D}(u))$ in view of (2) with $D$ replaced by $\hat{D}(u) D$, we finally obtain

$$
\psi\left(u(\hat{D}(w))^{2}\right)=u \psi\left((\hat{D}(w))^{2}\right)
$$

for all $u, w \in \mathcal{A}$. This shows that $\left[\mathcal{D}^{1}, \mathcal{A}\right]$ is a subset of the $\operatorname{radical} \operatorname{Rad}(\mathcal{J})$ of the ideal

$$
\mathcal{J}=\{v \in \mathcal{A}: \psi(u v)=u \psi(v), \forall u \in \mathcal{A}\} \subset \mathcal{A} \text {. }
$$

Now since $\mathcal{D}$ is non-singular, we have $\left[\mathcal{D}^{1}, \mathcal{A}\right]=\mathcal{A}$ so that $\operatorname{Rad}(\mathcal{J})$ and $\mathcal{J}$ both coincide with the whole algebra $\mathcal{A}$. The result follows immediately.

Definition $2([4,5,6])$. A quantum Poisson algebra $\mathcal{D}$ is symplectic if the constants are the only central elements.

Proposition 4. Let $\mathcal{D}_{1}$ and $\mathcal{D}_{2}$ be non-singular, symplectic and quasi-distinguishing quantum Poisson algebras. Any isomorphism $\Phi: \mathcal{D}_{1} \rightarrow \mathcal{D}_{2}$ respects the filtration and the restriction $\left.\Phi\right|_{\mathcal{A}_{1}}: \mathcal{A}_{1} \rightarrow \mathcal{A}_{2}$ is of the form

$$
\left.\Phi\right|_{\mathcal{A}_{1}}=k \Psi
$$

for some invertible element $k \in \mathcal{A}_{2}$, central in $\mathcal{D}_{2}$, and some isomorphism of $\mathbb{R}$-algebras $\Psi$ : $\mathcal{A}_{1} \rightarrow \mathcal{A}_{2}$. 
Proof. For all $D \in \mathcal{D}_{2}$ and all $u \in \mathcal{A}_{1}, w \in \mathcal{A}_{2}$, we have

$$
\left(\Phi \circ \gamma_{u} \circ \Phi^{-1}\right)([D, w])=\Phi\left[u \Phi^{-1}(D), \Phi^{-1}(w)\right]=\left[\Phi \circ \gamma_{u} \circ \Phi^{-1}(D), w\right] .
$$

In view of point 2) and point 3) of Proposition 3, it follows that

$$
\Phi\left(u \Phi^{-1}(w)\right)=\Phi\left(u \Phi^{-1}(1)\right) w \in \mathcal{A}_{2}
$$

so that $\Phi\left(u \Phi^{-1}(1)\right) \in \mathcal{A}_{2}$. As $\Phi^{-1}(1)$ is central in $\mathcal{D}_{1}$, it is a nonvanishing constant and thus $\Phi\left(\mathcal{A}_{1}\right) \subset \mathcal{A}_{2}$. Note that the same reasoning with $\Phi^{-1}$ yields $\Phi\left(\mathcal{A}_{1}\right)=\mathcal{A}_{2}$.

Now assume that $\Phi\left(\mathcal{D}_{1}^{i}\right) \subset \mathcal{D}_{2}^{i}$ for some $i \in \mathbb{N}$. For any $D \in \mathcal{D}_{1}^{i+1}$, we have

$$
\left[\Phi(D), \mathcal{A}_{2}\right]=\Phi\left(\left[D, \mathcal{A}_{1}\right]\right) \subset \mathcal{D}_{2}^{i}
$$

Using point 1) of Proposition 3, we obtain $\Phi(D) \in \mathcal{D}_{2}^{i+1}$ and thus $\Phi$ is filtered.

Finally, setting $v=\Phi^{-1}(w) \in \mathcal{A}_{1}$ and $\lambda=\Phi^{-1}(1)$, equality (3) used twice yields

$$
\Phi(u v)=\Phi(u \lambda) \Phi(v)=\Phi(\lambda u) \Phi(v)=\Phi\left(\lambda^{2}\right) \Phi(u) \Phi(v) .
$$

In particular, for $u=1$ and $v=\lambda$, the relation above shows that the element $\Phi\left(\lambda^{2}\right)$, central in $\mathcal{D}_{2}$, is also invertible in $\mathcal{A}_{2}$. Setting $\kappa=\Phi\left(\lambda^{2}\right)^{-1}$, the association

$$
u \in \mathcal{A}_{1} \mapsto \kappa^{-1} \Phi(u) \in \mathcal{A}_{2},
$$

defines an isomorphism of associative algebras and the conclusion is immediate.

Remark 1. The same property holds for isomorphisms between the Lie subalgebras $\mathcal{D}_{1}^{1}$ and $\mathcal{D}_{2}^{1}$. The proof is exactly the same.

Notice that for a vector bundle $E \rightarrow M$, the quantum Poisson algebra $\mathcal{D}_{\mathcal{E}}(E)$ is symplectic and quasi-distinguishing. These properties are easily shown from the local expression of the Poisson bracket in $\mathcal{S}_{\mathcal{E}}(E)$.

Corollary 1. Let $\pi: E \rightarrow M$ and $\eta: F \rightarrow N$ be two vector bundles. If $\Phi: \mathcal{D}_{\mathcal{E}}(E) \rightarrow \mathcal{D}_{\mathcal{E}}(F)$ is an isomorphism of Lie algebras, then $\Phi$ maps $\mathcal{A}(E)$ on $\mathcal{A}(F)$ and the restriction $\left.\Phi\right|_{\mathcal{A}}(E)$ : $\mathcal{A}(E) \rightarrow \mathcal{A}(F)$ reads

$$
\left.\Phi\right|_{\mathcal{A}(E)}=\kappa \Psi
$$

for some $\kappa \in \mathbb{R}_{0}$ and some isomorphism of $\mathbb{R}$-algebras $\Psi: \mathcal{A}(E) \rightarrow \mathcal{A}(F)$.

Remark 2. The same property holds for isomorphisms between the Lie subalgebras $\mathcal{D}_{\mathcal{E}}^{1}(E)$ and $\mathcal{D}_{\mathcal{E}}^{1}(F)$.

\section{Isomorphisms induce graded isomorphisms}

\subsection{Isomorphisms are filtered}

Lemma 1. Let $\pi: E \rightarrow M$ and $\eta: F \rightarrow N$ be two vector bundles. If $\Psi: \mathcal{A}(E) \rightarrow \mathcal{A}(F)$ is an isomorphism of $\mathbb{R}$-algebras, then

$$
\Psi\left(\mathcal{A}^{0}(E)\right)=\mathcal{A}^{0}(F) .
$$


Proof. For any nowhere vanishing $u \in \mathcal{A}^{0}(E)$, the function $u^{-1}: e \mapsto \frac{1}{u(e)}$ also belongs to $\mathcal{A}^{0}(E)$. Since $\Psi$ is a homomorphism,

$$
\mathcal{A}^{0}(F) \ni 1_{F}=\Psi\left(1_{E}\right)=\Psi\left(u \cdot u^{-1}\right)=\Psi(u) . \Psi\left(u^{-1}\right) .
$$

This implies that $\Psi(u)$ and $\Psi\left(u^{-1}\right)$ are both nonvanishing fiberwise constant polynomials since their product is a nonvanishing fiberwise constant polynomial.

Now, for any element $u \in \mathcal{A}^{0}(E), u^{2}+1_{E}: e \mapsto u(e)^{2}+1$ is a nonvanishing element in $\mathcal{A}^{0}(E)$. It follows that

$$
\mathcal{A}^{0}(F) \ni \Psi\left(u^{2}+1_{E}\right)=\Psi(u) \cdot \Psi(u)+1_{F},
$$

which shows that $\Psi(u)$ must be fiberwise constant.

We have proved $\Psi\left(\mathcal{A}^{0}(E)\right) \subset \mathcal{A}^{0}(F)$. The conclusion follows from applying the same arguments to the homomorphism $\Psi^{-1}$.

Lemma 2. Let $\left\{u_{1}, u_{2}, \ldots, u_{r}\right\} \cup \mathcal{A}^{0}(E)$ be a system of generators of the $\mathbb{R}$-algebra $\mathcal{A}(E)$. If $I \subset\{1,2, \ldots, r\}$ is the set of indices $i$ for which $u_{i}$ is of order at most 1 , then

$$
\left\{u_{i}: i \in I\right\} \cup \mathcal{A}^{0}(E)
$$

is again a system of generators of $\mathcal{A}(E)$.

Proof. It is obvious that the family (4) still generates $\mathcal{A}^{0}(E)$ and $\mathcal{A}^{1}(E)$. Since $\mathcal{A}^{0}(E)$ and $\mathcal{A}^{1}(E)$ generate the whole $\mathcal{A}(E)$, the lemma follows.

Proposition 5. Let $\pi: E \rightarrow M$ and $\eta: F \rightarrow N$ be two vector bundles. Every isomorphism of $\mathbb{R}$-algebras $\Psi: \mathcal{A}(E) \rightarrow \mathcal{A}(F)$ is filtered with respect to the filtrations of $\mathcal{A}(E)$ and $\mathcal{A}(F)$ associated with their gradings.

Proof. Since degrees 0 and 1 generate the whole $\mathbb{R}$-algebras, it is enough to show

$$
\Psi\left(\mathcal{A}^{0}(E)\right) \subset \mathcal{A}^{0}(F), \quad \Psi\left(\mathcal{A}^{1}(E)\right) \subset \mathcal{A}^{1}(F) \oplus \mathcal{A}^{0}(F) .
$$

The first relation comes from Lemma 1. Let us prove the second one.

The $\mathrm{C}^{\infty}(M)$-module $\Gamma\left(E^{*}\right)$ of global sections of the dual bundle $\pi^{*}$ is (projective and) finitely generated. Let $\left\{\alpha_{1}, \alpha_{2}, \ldots, \alpha_{r}\right\}$ be a system of generators. Passing through the isomorphism $\Gamma\left(E^{*}\right) \cong \mathcal{A}^{1}(E)$ and adding fiberwise constant polynomials, we obtain a set of generators

$$
\left\{u_{1}, u_{2}, \ldots, u_{r}\right\} \cup \mathcal{A}^{0}(E)
$$

for the $\mathbb{R}$-algebra $\mathcal{A}(E)$. Since $\Psi$ is an isomorphism, the images

$$
\left\{\Psi\left(u_{1}\right), \Psi\left(u_{2}\right), \ldots, \Psi\left(u_{r}\right)\right\} \cup \mathcal{A}^{0}(F)
$$

generate the whole $\mathcal{A}(F)$. In view of Lemma 2, this set of generators can be refined into

$$
\left\{\Psi\left(u_{i}\right): i \in I\right\} \cup \mathcal{A}^{0}(F),
$$

where $I \subset\{1,2, \ldots, r\}$ is the set of indices for which $\Psi\left(u_{\alpha_{i}}\right) \in \mathcal{A}^{1}(F) \oplus \mathcal{A}^{0}(F)$. Since $\Psi^{-1}$ is an isomorphism, every element in $\mathcal{A}^{1}(E)$ can now be decomposed as an $\mathbb{R}$-linear combination of the generating family

$$
\left\{u_{i}: i \in I\right\} \cup \mathcal{A}^{0}(E) .
$$

The conclusion is immediate. 


\subsection{Isomorphisms induce graded isomorphisms}

For $k \in \mathbb{N}$, denote by $\operatorname{pr}_{k}$ the projection of fiberwise polynomial functions onto their homogeneous component of order $k$.

Proposition 6. If $\Psi: \mathcal{A}(E) \rightarrow \mathcal{A}(F)$ is an isomorphism of $\mathbb{R}$-algebras, then for any $k \in \mathbb{N}$, the map

$$
\widetilde{\Psi}_{k}=\left.\operatorname{pr}_{k} \circ \Psi\right|_{\mathcal{A}^{k}(E)}: \mathcal{A}^{k}(E) \rightarrow \mathcal{A}^{k}(F)
$$

is a linear bijection.

Proof. First, $\widetilde{\Psi}_{k}$ is surjective. Given $w \in \mathcal{A}^{k}(F)$, there is an $u \in \mathcal{A}^{k}(E) \oplus \cdots \oplus \mathcal{A}^{1}(E) \oplus \mathcal{A}^{0}(E)$ such that $w=\Psi(u)$. Using the fact $\Psi$ is filtered (in view of Proposition 5), it comes

$$
\mathcal{A}^{k}(F) \ni w=\operatorname{pr}_{k} \circ \Psi(u)=\operatorname{pr}_{k} \circ \Psi\left(\operatorname{pr}_{k}(u)\right) \in \widetilde{\Psi}_{k}\left(\mathcal{A}^{k}(E)\right) .
$$

Next, $\widetilde{\Psi}_{k}$ is also injective. If $u \in \mathcal{A}^{k}(E)$ satisfies $\operatorname{pr}_{k} \circ \Psi(u)=0$, then $\Psi(u) \in \mathcal{A}^{k-1}(F) \oplus \cdots \oplus$ $\mathcal{A}^{1}(F) \oplus \mathcal{A}^{0}(F)$. Since $\Psi^{-1}$ is filtered (in view of Proposition 5), we get

$$
\mathcal{A}^{k}(E) \ni u=\Psi^{-1}(\Psi(u)) \in \mathcal{A}^{k-1}(E) \oplus \cdots \oplus \mathcal{A}^{1}(E) \oplus \mathcal{A}^{0}(E) .
$$

Thus $u=0$.

Corollary 2. If $\Psi: \mathcal{A}(E) \rightarrow \mathcal{A}(F)$ is an isomorphism of $\mathbb{R}$-algebras, then the map $\widetilde{\Psi}: \mathcal{A}(E) \rightarrow$ $\mathcal{A}(F)$ whose restriction to each $\mathcal{A}^{k}(E)$ is given by $\widetilde{\Psi}_{k}$ is a graded isomorphism of $\mathbb{R}$-algebras.

Proof. The map $\tilde{\Psi}$ is obviously bijective, linear and graded. Moreover, it is a homomorphism of $\mathbb{R}$-algebras. Indeed, for any $u_{1} \in \mathcal{A}^{k_{1}}(E)$ and $u_{2} \in \mathcal{A}^{k_{2}}(E)$,

$$
\begin{aligned}
\widetilde{\Psi}\left(u_{1} u_{2}\right) & =\operatorname{pr}_{k_{1}+k_{2}} \circ \Psi\left(u_{1} u_{2}\right)=\operatorname{pr}_{k_{1}+k_{2}}\left(\Psi\left(u_{1}\right) \Psi\left(u_{2}\right)\right) \\
& =\left(\operatorname{pr}_{k_{1}} \circ \Psi\left(u_{1}\right)\right)\left(\operatorname{pr}_{k_{2}} \circ \Psi\left(u_{2}\right)\right)=\widetilde{\Psi}\left(u_{1}\right) \widetilde{\Psi}\left(u_{2}\right),
\end{aligned}
$$

where we have used the fact that the highest order terms of the product of fiberwise polynomial functions is the product of their highest order terms.

\section{A Lie algebraic characterization}

After a technical lemma, we will be in a position to establish a new Pursell-Shanks type result for vector bundles, in terms of the Lie algebra generated by differential operators that are eigenvalues of the Lie derivative in the direction of the Euler vector field associated with each vector bundle.

Lemma 3. If $0=j_{a}^{l} u, u \in \mathcal{A}$, then

$$
u=\sum_{i=1}^{N} u_{i_{0}} \cdots u_{i_{l}}
$$

where $u_{i_{j}} \in \mathcal{A}$ and $u_{i_{j}}(a)=0, \forall(i, j) \in[1, N] \times[0, l]$.

Proof. Consider $u \in \mathcal{A}$ such that $j_{a}^{l}(u)=0$ and $U$ a domain of trivialization such that $\pi(a) \in U$. One has over $U$,

$$
\frac{d^{l+1}}{d t^{l+1}} u(a+t h)=\sum_{i_{0}, \ldots, i_{l}}\left(\partial_{i_{0} \ldots i_{l}} u\right)(a+t h) h^{i_{0}} \cdots h^{i_{l}} .
$$


From this, it may be deduced by successive primitivations in $[0, t]$

$$
u(x)=\sum_{i_{1}, \ldots, i_{l}}\left(x^{i_{0}}-a^{i_{0}}\right) \cdots\left(x^{i_{l}}-a^{i_{l}}\right) v(x)
$$

where we put $x=a+t h$. In the right member of this equality, factors are local, but observe that one can write

$$
u=\left(1-\alpha^{l+1}\right) u+\alpha^{l+1} u
$$

with $\alpha=\pi^{*}\left(\alpha^{\prime}\right)$ where $\alpha^{\prime} \in \mathrm{C}^{\infty}(M, \mathbb{R})$ equals to 1 in a neighborhood $V \subset U$ containing $\pi(a)$ and with compact support in $U$. The term $\alpha^{l+1} u$ becomes

$$
\sum_{i_{1}, \ldots, i_{l}} \alpha\left(x^{i_{0}}-a^{i_{0}}\right) \cdots \alpha\left(x^{i_{l}}-a^{i_{l}}\right) v(x)
$$

and has the form (5). For the other term, one proceeds in the same way by using the fact that

$$
1-\alpha^{l+1}=\left(1-\alpha^{l+1}\right)(1-\beta)^{l+1},
$$

with $\beta=\pi^{*}\left(\beta^{\prime}\right)$ where $\beta^{\prime} \in \mathrm{C}^{\infty}(M, \mathbb{R})$ is of compact support in a neighborhood $W \subset V$ containing $\pi(a)$, and in which it is equal to 1 .

Corollary 3. For all $D \in \operatorname{Der}(\mathcal{A})$ there exists an unique differential operator $\widehat{D} \in \mathcal{D}^{1}(E, M)$ such that

$$
\widehat{D}(u)=D(u) \quad \forall u \in \mathcal{A} .
$$

Proof. We use a most general result stating that the corollary is true for a linear map $D: \mathcal{A} \rightarrow \mathcal{A}$ which depends only on 1-jets of its arguments (in the sense that $D$ vanishes on arguments of zero 1-jet). Indeed, this statement results from the fact that for any function $f \in \mathrm{C}^{\infty}(E, \mathbb{R})$, any point $a \in E$ and any integer $k \in \mathbb{N}$, there exists $u \in \mathcal{A}$ such that

$$
j_{a}^{k}(f)=j_{a}^{k}(u)
$$

hence one can write

$$
\widehat{D}(f)(a)=D(u)(a) .
$$

Considering $D \in \operatorname{Der}(\mathcal{A})$ and $u \in \mathcal{A}$ such that $j_{a}^{1}(u)=0$, one has

$$
D(u)(a)=0 .
$$

Indeed, with the notations of Lemma 3, one has

$$
D(u)=\sum_{i=1}^{N} u_{i_{0}} D\left(u_{i_{1}}\right)+\sum_{i=1}^{N} u_{i_{0}} D\left(u_{i_{1}}\right) .
$$

Therefore $D(u)$ vanishes at $a$. 


\subsection{The main result}

The proof of the main result will rely on a theorem of [7], which we shall recall before stating out main result. As this theorem requires some hypotheses on both the base manifold and the rank of the vector bundles, our result will inherit those hypotheses.

Theorem 1 ([7]). Let $\pi: E \rightarrow M$ and $\eta: F \rightarrow N$ be two vector bundles of ranks $n, n^{\prime}>1$. If the first space $\mathrm{H}^{1}(M, \mathbb{Z} / 2)$ of the Cech cohomology of $M$ vanishes, then the two vector bundles are isomorphic if and only if the Lie algebras $\operatorname{Aut}(E)$ and $\operatorname{Aut}(F)$ of infinitesimal automorphisms are isomorphic.

Theorem 2. Under the same hypotheses as in Theorem 1, two vector bundles $\pi: E \rightarrow M$ and $\eta: F \rightarrow N$ are isomorphic if and only if the Lie algebras $\mathcal{D}_{\mathcal{E}}(E)$ and $\mathcal{D}_{\mathcal{E}}(F)\left(\right.$ resp. $\mathcal{D}_{\mathcal{E}}^{1}(E)$ and $\left.\mathcal{D}_{\mathcal{E}}^{1}(F)\right)$ are isomorphic.

Proof. First, note that if $\Psi: \mathcal{A}(E) \rightarrow \mathcal{A}(F)$ is an isomorphism of graded $\mathbb{R}$-algebras $\mathcal{A}(E) \rightarrow$ $\mathcal{A}(F)$, the induced isomorphism $\widehat{\Psi}: \operatorname{Der}(\mathcal{A}(E)) \rightarrow \operatorname{Der}(\mathcal{A}(E))$ transforms 0-weight derivations of $\mathcal{A}(E)$ into 0 -weight derivations of $\mathcal{A}(F)$. Then, the theorem is a consequence of Theorem 1 , if we prove that $\operatorname{Der}^{0}(\mathcal{A}(E))=\left.\operatorname{Aut}(E)\right|_{\mathcal{A}(E)}$. On the one hand, the inclusion $\operatorname{Der}^{0}(\mathcal{A}(E)) \supset$ $\left.\operatorname{Aut}(E)\right|_{\mathcal{A}(E)}$ is obvious. On the other hand, for $D \in \operatorname{Der}^{0}(\mathcal{A}(E))$, Corollary 3 yields $\widehat{D} \in$ $\operatorname{Vect}(E)$ such that $D=\left.\widehat{D}\right|_{\mathcal{A}(E)}$. As $\left.\left[\mathcal{E}_{E}, \widehat{D}\right]\right|_{\mathcal{A}(E)}=0$, it follows that $\left[\mathcal{E}_{E}, \widehat{D}\right]=0$.

Remark 3. Along the way, we have also proved that under the same hypotheses as above, two vector bundles $\pi: E \rightarrow M$ and $\eta: F \rightarrow N$ are isomorphic if and only if the $\mathbb{R}$-algebras $\mathcal{S}_{\mathcal{E}}(E)$ and $\mathcal{S}_{\mathcal{E}}(F)$ (resp. $\mathcal{S}_{\mathcal{E}}^{1}(E)$ and $\mathcal{S}_{\mathcal{E}}^{1}(F)$ ) are isomorphic. Finally, note that without any hypothesis, Corollary 1 and Lemma 1 ensure in view of Milnor's theorem that any isomorphism between $\mathcal{D}_{\mathcal{E}}(E)$ and $\mathcal{D}_{\mathcal{E}}(F)$ induces a diffeomorphism between the base manifolds $M$ and $N$.

\section{Acknowledgements}

We thank the referees for suggestions leading to improvements of the original paper.

\section{References}

[1] Gel'fand I.M., Kolmogorov A.N., On rings of continuous functions on topological spaces, Dokl. Akad. Nauk SSSR 22 (1939), no. 1, 7-10.

[2] Grabowski J., Kotov A., Poncin N., Lie superalgebras of differential operators, arXiv:1011.1804.

[3] Grabowski J., Kotov A., Poncin N., The Lie superalgebra of a supermanifold, J. Lie Theory 20 (2010), 739-749.

[4] Grabowski J., Poncin N., Automorphisms of quantum and classical Poisson algebras, Compos. Math. 140 (2004), 511-527, math.RA/0211175.

[5] Grabowski J., Poncin N., Lie algebraic characterization of manifolds, Cent. Eur. J. Math. 2 (2004), 811-825, math.DG/0310202.

[6] Grabowski J., Poncin N., On quantum and classical Poisson algebras, in Geometry and Topology of Manifolds, Banach Center Publ., Vol. 76, Polish Acad. Sci., Warsaw, 2007, 313-324, math.DG/0510031.

[7] Lecomte P., On the infinitesimal automorphisms of a vector bundle, J. Math. Pures Appl. (9) 60 (1981), $229-239$.

[8] Shanks M.E., Pursell L.E., The Lie algebra of a smooth manifold, Proc. Amer. Math. Soc. 5 (1954), 468-472. 